\title{
Repaso de las propuestas para el desarrollo de América Latina y el Caribe
}

\author{
Jorge Mario Martínez Piva'
}

Resumen: Este artículo repasa el desarrollo de América Latina y el Caribe de los últimos 70 años a partir de la experiencia y propuestas que ha hecho la Comisión Económica para América Latina y el Caribe (CEPAL). Se analiza la relación centro periferia y la propuesta de industrialización por sustitución de importaciones, la crisis de esta propuesta y el subsiguiente modelo basado en el regionalismo abierto. La crisis de 2008 parece un punto de inflexión a la apertura comercial como modelo de desarrollo y se abre un periodo de búsqueda con énfasis en la sostenibilidad y la igualdad.

Palabras clave: desarrollo, industrialización, regionalismo abierto, desarrollo sostenible.

\section{Review of proposals for the development of Latin America and the Caribbean}

Abstract: This article reviews the development of Latin America and the Caribbean over the last 70 years, based on the experience and proposals made by the Economic Commission for Latin America and the Caribbean. It analyses the centre-periphery relationship and the proposal of import substitution industrialisation, the crisis of this proposal and the subsequent model based on open regionalism. The 2008 crisis seems to be a
Révision des propositions pour le développement de l'Amérique Latin et les Caraibes

Résumé: Cet article faitle point sur le développement de l'Amérique Latine et les Caraïbes au cours des 70 dernières années, sur la base de l'expérience et des propositions de la Commission Économique pour l'Amérique Latine et les Caraibes. Il analyse la relation centre-périphérie et la proposition d'industrialisation par substitution aux importations, la crise de cette proposition et le modèle ultérieur basé sur le régionalisme ouvert. La

' Comisión Económica para América Latina y el Caribe. 
turning point for open trade as a development model, and a period of search opens up with an emphasis on sustainability and equality.

Key words: development, industrialisation, open regionalism, sustainable development. crise de 2008 semble être un tournant pour le commerce ouvert en tant que modèle de développement, et une période de recherche s'ouvre avec un accent sur la durabilité et l'égalité.

Mots clé: développement, industrialisation, régionalisme ouvert, développement durable.

\section{Introducción: desarrollo como cambio estructural}

Este artículo repasa el desarrollo de América Latina y el Caribe de los últimos 70 años a partir de las propuestas que ha hecho la Comisión Económica para América Latina y el Caribe (CEPAL). La CEPAL ha generado desde su fundación en 1948 un andamiaje analítico sobre el desarrollo de la región y un conjunto de políticas públicas para lograrlo. Desde entonces los trabajos de la CEPAL han sido de "continuidad con cambios" (Bárcena y otros, 2018), ajustándose a los avances intelectuales, metodológicos y al entorno internacional.

El método analítico de la CEPAL es el histórico-estructural, que subraya la relevancia del contexto histórico y social para entender el funcionamiento de la economía. Las teorías y leyes económicas generales no pueden captar las circunstancias históricas y sociales que explican el funcionamiento de una economía. Por el contrario, en el método histórico-estructural los aspectos sociales y políticos circunscritos en periodos históricos y sociedades concretas, son fundamentales para entender el funcionamiento de la economía en y ese sentido se acerca al análisis clásico de la economía política.

El enfoque estructuralista subraya la relación entre estructura productiva y los cambios necesarios para lograr el desarrollo económico. Este implica no sólo altas tasas de crecimiento sino también cambios cualitativos en la estructura productiva, en el empleo, en el patrón de especialización e inserción en la economía mundial. Por esta razón se afirma que el desarrollo implica un cambio estructural que debe conducir a la superación de la condición de periferia y de las características estructurales que limitan el crecimiento de largo plazo: una estructura productiva basada en bienes primarios con poco valor agregado y una baja diversificación productiva, con escasa integración vertical en el tejido productivo; poca integración entre la dinámica industrial y agrícola que afecta la productividad, la innovación 
y permite mayor acumulación de ganancias en el sector secundario; bajos niveles de innovación; alta heterogeneidad de la productividad entre sectores ${ }^{2}$ y alta desigualdad del ingreso; bajos niveles de inversión nacional relacionado con los bajos niveles de ahorro; concentración de las exportaciones por destinos y por productos generando vulnerabilidad a los choques externos (Sánchez Díez y Martínez Piva, 2014).

América Latina y el Caribe ha transitado desde de la década de los 50 tres grandes propuestas de pwolíticas para el desarrollo: la primera, para superar la fase exportadora de productos primarios fue la industrialización por sustitución de importaciones (modelo de desarrollo hacia adentro); la segunda, la integración con la economía global al tiempo que se fortalecía la integración regional (el regionalismo abierto); y la tercera, recientemente propuesta por la CEPAL, por una transformación productiva con énfasis en la igualdad (cambio estructural progresivo).

Este artículo se divide en esta introducción, una sección sobre la industrialización para el cambio estructural, otra sobre la integración a la economía global y finalmente una sección sobre una nueva estrategia para el cambio estructural que atienda las crisis actuales. A modo de conclusión, las reflexiones finales abordan los éxitos parciales de las estrategias de desarrollo propuestas y la importancia de su renovación.

\section{Industrialización para el cambio estructural}

La América Latina y el Caribe de los años 50 era fundamentalmente rural, especializada en bienes primarios, poco diversificados y con poco valor agregado. La estructura productiva heterogénea y la alta concentración de la propiedad contribuían a la concentración del ingreso. Bajos niveles de inversión, y bajos niveles de investigación y desarrollo impedían el cambio de dicha estructura productiva, a lo que se sumaba un contexto internacional que la profundizaba. La alta participación de la inversión extranjera en actividades extractivas con baja propensión a invertir en actividades de innovación y desarrollo, así como la concentración de las exportaciones en pocos productos y pocos mercados de destino hacía que la economía fuera dependiente y vulnerable a los choques externos. Además, los países latinoamericanos enfrentaban una constante res-

${ }^{2}$ Aníbal Pinto denominaba heterogeneidad estructural a la existencia de sectores económicos con niveles de productividad muy diferentes. Pinto, 1970. 
tricción externa: cuando los precios de los bienes de exportación eran altos, se incrementaban las importaciones y se afectaba negativamente la balanza de pagos, impidiendo el ahorro y la inversión.

Las características anteriores explican la relación de centro-periferia que los países latinoamericanos mantienen con los desarrollados - del centro- y que se caracteriza por los mayores beneficios económicos que los países del centro obtienen y la condición perpetua de periferia de los países latinoamericanos.

Los análisis sobre la relación centro-periferia se enfocaron en el deterioro de los términos de intercambio entre los países de la periferia en relación con los países del centro. Este deterioro era resultado de la especialización de los países periféricos en bienes primarios, cuyo valor se deterioraba respecto de sus importaciones de los países del centro, especializados en bienes industriales con mayor contenido tecnológico y cuyo valor relativo incrementaba con el tiempo. En el proceso de creación de valor de la producción y de su apropiación, el contenido tecnológico de los bienes industriales juega un papel muy importante por lo que a mediados del siglo XX la CEPAL propuso una estrategia de desarrollo enfocada en superar la fase agroexportadora que promovía la industrialización mediante la sustitución de importaciones.

Desde la década de 1950 América Latina y el Caribe diseñó políticas para su industrialización y a la sustitución de importaciones. El Estado asumió un rol fundamental rompiendo las inercias de una economía basada en recursos naturales y bienes primarios que reproducía un modelo social en el que lo rural y la concentración de la propiedad productiva eran una constante. Para promover la industrialización, se protegieron las industrias nacientes con altos aranceles, cuotas y restricciones sectoriales a la inversión extranjera o cláusulas de desempeño, entre otras políticas. En muchas ocasiones el Estado asumió la producción de bienes esenciales para el proceso de industrialización: hubo inversiones a gran escala en infraestructura de transportes y energética, así como en la urbanización acelerada debido a la concentración de los nuevos empleos en las ciudades (Bulmer-Thomas, 2003; Martínez Piva, 2015; Moreno-Brid et al., 2004).

Tras años de rápido crecimiento y cambios notorios en la composición del producto, de la urbanización y extensión de los servicios públicos básicos, en la década de 1970 el modelo de industrialización por sustitución de importaciones empezó a dar signos de agotamiento. Los principales elementos que condujeron al declive de la estrategia tienen que ver con que no se superó la dependencia de insumos extranjeros, de productos intermedios y de innovación. Además, se generaron pocos 
encadenamientos productivos y mantuvieron grandes diferencias de productividad entre sectores al interior de los países y con sectores en rubros similares en los países del centro. El esfuerzo de transformación estructural condujo a un acelerado crecimiento de los servicios públicos al tiempo que se mantuvo una estructura fiscal ineficiente y regresiva. Esa fragilidad fiscal y el amplio uso de la deuda externa para financiar el esfuerzo desarrollista condujeron a desequilibrios en la balanza de pagos y en las cuentas fiscales. El modelo colapsó ante los choques externos generados por el alza de los precios del petróleo y de los intereses de la deuda externa (Bulmer-Thomas, 2003; Moreno-Brid et al., 2004).

\section{De la globalización a la necesidad de un crecimiento sostenible}

La crisis de la deuda externa a inicios de la década de 1980 condujo al cuestionamiento del rol desarrollista del Estado por un grupo influyente de economistas y políticos defensores del libre mercado y de la reducción del papel del Estado. Este grupo propuso políticas con énfasis en medidas macroeconómicas de estabilización -reducción del déficit fiscal, control de la inflación, balance de la cuenta externa y pago de la deuda, reducción del tamaño del Estado y de su rol en la economía. Los defensores de los méritos del libre mercado y de las restricciones del Estado en la economía constituyeron el llamado "Consenso de Washington".

América Latina y el Caribe ajustó sus políticas económicas a las nuevas propuestas, algunas impuestas por sus acreedores, así como por nuevos ideólogos del desarrollo. Los principales objetivos de la nueva estrategia fueron la estabilización de las debilitadas cuentas públicas, obtención de recursos financieros para enfrentar las obligaciones del Estado y promover que el mercado guiara las inversiones para el crecimiento (Sunkel, 1991; Martínez Piva, 2001 ; Bulmer-Thomas, 2003; Ortiz, 2003; Moreno-Brid et al., 2004). Se impulsó la liberalización financiera, la apertura a la inversión extranjera, la privatización de empresas estatales y la inserción en los mercados internacionales, contando con que éstos facilitarían el acceso a mercados dinámicos y al conocimiento tecnológico (Padilla-Pérez y Martínez-Piva, 2009). La mayoría de los instrumentos para fomentar la industrialización fueron eliminados. Desaparecieron los subsidios al crédito productivo, se abandonaron los programas de desarrollo sectoriales, y se favorecieron las políticas horizontales -educación, infraestructura, facilitación de trámites, etc.-, se redujo la inversión pública, tanto la productiva como la de apoyo a la producción, se redujeron los aranceles y se promovió la competencia (Martínez Piva, 2015). 
La CEPAL promovió el regionalismo abierto, ajustando la estrategia de crecimiento e industrialización y balanceando el exceso de aperturismo propuesto por el Consenso de Washington. Se propuso hacer compatibles y complementarias las políticas de competencia internacional con la integración regional (CEPAL, 1994). Se siguió señalando la industrialización como necesaria, pero para superar tanto las estrategias de desarrollo hacia adentro como las propuestas unilaterales de liberalización, era necesario no prejuzgar a favor de la sustitución de importaciones.

La propuesta de regional ismo abierto apuntó hacia la industrialización desde dentro, basada en industrias pilares como núcleo endógeno que permitiera la generación, acumulación y difusión del progreso técnico. La propuesta intentaba superar las estrategias de desarrollo hacia adentro, así como las propuestas unilaterales de crecimiento hacia afuera. La idea se fundamentaba en aprovechar el dinamismo de la demanda externa -exportaciones-, el flujo de capitales -inversión extranjera-, se fomentaba el mercado regional -integración-y se fortalecían industrias pilares como núcleo endógeno de la industrialización (Sunkel, 1991).

La mayoría de los países de la reigón aplicaron estrategias comerciales en las que conjugaban el aprovechamiento del mercado regional (renovación de los mecanismos de integración regional) y la mejora de la inserción internacional a través de diversos acuerdos de libre comercio.

La consolidación del mercado europeo y su proyecto de integración regional puso de relieve las ventajas que la integración podía aportar a sus miembros y cómo ese proceso podía ser funcional para su inserción internacional. El Tratado de Libre Comercio de América del Norte confirmó esa percepción... (Martínez Piva, 2019, pp. 71).

Estos cambios se potenciaron gracias a tecnologías emergentes, sobre todo las tecnologías de la comunicación, las que sumadas al abaratamiento relativo del transporte, hicieron posible la creación de procesos productivos deslocalizados. La reorganización de la producción global, con el consecuente crecimiento del comercio y de los flujos financieros internacionales culminó el proceso bautizado como "globalización".

Los mecanismos de integración regional se renovaron. En Centroamérica, el proceso de integración aprobó en 1990 el Plan de Acción Económico en Centroamérica (PAECA), con el fin de crear un nuevo marco jurídico, una nueva estructura arancelaria, analizar la política exterior conjunta, evolucionar de una zona de libre comercio a una comunidad económica, entre otras acciones. En 1991 Costa Rica, El Salvador, Guatemala, Honduras, Nicaragua y Panamá suscribieron el Protocolo de Tegucigalpa, en el que se estableció y consolidó el Sistema de Integración Cen- 
troamericana $(\mathrm{SICA})^{3}$. Panamá se incorporó como Estado Miembroy posteriormente se adhirieron Belice y la República Dominicana. La Comunidad del Caribe (CARICOM, Caribbean Community) revisó en 2002 su tratado fundacional de 1973 para permitir el eventual establecimiento de un mercado único y una economía única, y creó la Corte Caribeña de Justicia. La Comunidad Andina constituyó en 1990 el Consejo Presidencial Andino; en 1991 se ratificaron los acuerdos para institución del arancel externo común y determinó la armonización de políticas cambiarias y monetarias $y$, se definieron las políticas externas del grupo, que culminó en tratativas comerciales con los países miembros del MERCOSUR. En 1993 se creó la Zona Andina de Libre Comercio y se instituyó el arancel externo común y en 1996 se fortaleció el proceso de integración con varias reformas a partir de las cuales el Acuerdo de Cartagena que dio origen al Pacto Andino tomó el nombre de Comunidad Andina.

En 1997, el bloque experimenta un giro institucional y estructural (...) la integración pasa a ser no apenas técnica y comercial, sino también de contenido claramente político, al insertar en su estructura el Consejo Presidencial Andino y el Consejo Andino de Ministros de RR. EE., además de convertir la Junta del Acuerdo de Cartagena en un Secretaría General con funciones políticas, originando, así, el Sistema Andino de Integración y la Comunidad Andina en reemplazo del Pacto Andino (Contipelli, 2016, pp. 265).

El Mercado Común del Sur (MERCOSUR) es el proceso de integración regional más joven y es parte del impulso renovado por la integración regional en la década de 1990. Se funda a través del Tratado de Asunción en marzo de 1991 y en 1994, el Protocolo de Ouro Preto crea la estructura institucional del MERCOSUR. Su objetivo fundacional es la creación de un Mercado Común, previsto para diciembre de 1994, y que debería permitir la libre circulación de bienes, servicios y factores productivos entre los países socios.

El comercio internacional y los flujos de inversión extranjera directa (IED) aumentaron de manera significativa. Las exportaciones de los países en desarrollo crecieron a una tasa promedio de $8,4 \%$ entre 2000 y 2018 mientras que el mundo en su conjunto lo hizo al 6,4\% y las economías desarrolladas a 5\%. La participación de las economías en desarrollo en el en la recepción de flujos de IED también se incrementó notablemente de 17\% en 1990 a 44,4\% del total en 2019.

El proceso liberalizador de la década de 1980 pero acelerado en la de 1990 tuvo gran impacto en la estructura productiva global y en la de muchos países en

${ }^{3}$ Como resultado de la transformación de la Organización de los Estados Centroamericanos (ODECA) creada en 1951. Su función principal sería dar seguimiento a las decisiones de la Reunión de Presidentes y coordinar la ejecución de las mismas a través de las diferentes instituciones regionales existentes 
desarrollo. Los países centroamericanos y México, por ejemplo, pasaron de tener una canasta exportadora en la que el $58,7 \%$ para los primeros y $48,3 \%$ para el segundo, eran bienes primarios a principios de la década de los noventa, a una estructura exportadora en 2019 en la que los bienes primarios son solo el 28,7\% de las exportaciones totales centroamericanas y el 10,2\% de las mexicanas. Este cambio tiene que ver en gran medida con su vinculación a diversas cadenas globales de valor.

El modelo de inserción internacional que siguió la región de América Latina y el Caribe fue acompañado por un conjunto de políticas macroeconómicas con enfoque estabilizador. Centraron sus objetivos en controlar la inflación y el déficit fiscal con resultados, en términos generales, positivos. La inflación en la región entre 1990 y 2018 fue en promedio de 6,3\%, lejos de los procesos de hiperinflación de la década de 1980; la deuda pública bruta pasó de 60\% del PIB en 1990 a menos del $40 \%$ en 2017.

Sin embargo, esta propuesta de desarrollo mostró debilidades que se hicieron evidentes tras la crisis de 2008. El PIB per capita de América Latina no ha logrado reducir la brecha que le separa con los países más ricos. En 1990 el PIB per capita promedio de América Latina y el Caribe era el $15 \%$ del de Estados Unidos y para 2018 ese porcentaje era el 14\%. El crecimiento de la productividad laboral ha sido magro y nunca alcanzó los niveles de obtenidos en el periodo de industrialización por sustitución de importaciones. Mientras que la productividad laboral de Argentina, Brasil y México crecieron en promedio 1,1, 3,7 y 1,8 entre 1965 y 1981 respectivamente, esta creció solo 0,4, 1,3 y 0,06 entre 1982 y 2010 . En estos mismos periodos la productividad laboral de la República de Corea creció al 4,7 y 4,3 lo que subraya el enorme reto que tiene América Latina y el Caribe en materia políticas productivas (CEPAL, 2012).

Por otra parte, el aumento de la desigualdad en las principales economías del mundo es un factor característico del periodo de apertura y globalización, lo que ha conducido al aumento de las tensiones políticas internas y a conflictos comerciales. Desde una perspectiva de largo plazo, entre 1980 y 2016 , el $1 \%$ más rico de la población mundial aumentó sus ingresos de manera constante en la mayoría de los países y capturó el $27 \%$ del crecimiento acumulado total, mientras que el $50 \%$ más pobre capturó solo el 12\%. (CEPAL, 2020). Lamentablemente América Latina y el Caribe es la región más desigual del mundo, por lo que cualquier estrategia de desarrollo debe atender en este problema. La desigualdad no es solo un asunto de ingresos, sino también de derechos, de acceso a la sanidad a la educación, de igualdad de género, acceso al saneamiento, a la vivienda y a la movilidad para 
grandes grupos de la población. El 20\% de los habitantes urbanos en América Latina lo hace en asentamientos precarios, que son una manifestación física de la desigualdad. Es necesario hacer correcciones en la estrategia de desarrollo para atender la desigualdad social.

Finalmente, la propuesta de desarrollo basada en la globalización económica no supo enfrentar la crisis del calentamiento global, que pone en riesgo, no solo a la economía, sino a la supervivencia del planeta como se conoce hoy. Diversas ciencias han incorporado en sus análisis las previsiones de la crisis ambiental y hoy existe abundante literatura económica sobre este problema y lo incorpora al análisis del crecimiento y el desarrollo (Nordhaus, 1994; Stern, 2007; Dasgupta, 2021 ) concluyendo que el calentamiento global es la mayor amenaza que enfrenta la humanidad y que debe ser atendida con urgencia.

Si bien los países de latinoamericanos y caribeños no son grandes emisores de gases de efecto invernadero (en 2017, poco más del $8 \%$ de las emisiones mundiales de GEl se generaron en América Latina y el Caribe: 4,3 de 51 gigatoneladas de $\mathrm{CO} 2$ eq.), estos países observan un creciente deterioro de sus recursos naturales y crecientes amenazas de los eventos meteorológicos extremos cuyo origen es el calentamiento global. Además, en la región las emisiones han aumentado en casi todos los sectores, siendo el sector de la energía es el que más emite y cuyas emisiones más han aumentado (CEPAL, 2020).

En América Latina y el Caribe la crisis ambiental se expresa en incrementos en la temperatura promedio de gran parte de la región de al menos un grado superior al promedio, una elevación del mar equivalente a unos 15 centímetros, e incrementos de eventos extremos como huracanes, y sequías prolongadas. Algunas regiones como la centroamericana y la caribeña tienen índices muy altos de riesgos y están viviendo pérdidas importantes que tienden a incrementarse con el tiempo. Según el Índice de Riesgo Mundial, que muestra el nivel de exposición y la vulnerabilidad a los fenómenos climáticos extremos, cuatro países de la región (Dominica, Haití, Honduras y Nicaragua) están entre los diez más afectados del mundo en los últimos 20 años, tanto por fenómenos excepcionalmente devastadores como por la continuidad de estos (Eckstein y otros, 2019, citado en CEPAL, 2020).

La CEPAL ha estimado que en Centroamérica la precipitación media mensual puede reducirse entre el 11 y el 28\% para finales de este siglo, según el escenario de calentamiento previsto, A2 ○ B2. También ha estimado que la producción de granos básicos como el maíz, el frijol y el arroz podría reducir su rendimiento (toneladas/hectárea) hasta en $35 \%, 43 \%$ y $50 \%$ respectivamente en un escenario A2 (CEPAL y otros, 2015). 
En los escenarios climáticos del IPCC... Tanto Centroamérica como el Caribe serán más cálidos y secos. Otros efectos negativos serán el alza del nivel del mar, que acentúa la pérdida y la erosión de las áreas costeras, y el deterioro de los ecosistemas marinos, particularmente de los arrecifes de coral. Los costos humanos y económicos de los desastres relacionados con el clima son cuantiosos. Entre 1990 y 2018, los daños ocurridos en el Caribe representaron un costo total de 140.000 millones de dólares. En cinco años, 1998, 2004, 2010, 2016 y 2017, los daños costaron más de 5.000 millones de dólares. En ese conjunto de años ocurrió el $87,2 \%$ de la destrucción de activos de todo el período. La temporada de huracanes de 2017 representó el 66,7\% del total de daños del período y tuvo un costo de 93.500 millones de dólares. En dicha temporada, el costo total de los huracanes Irma y María en las Islas Vírgenes Británicas y San Martín duplicó con creces el PIB de esos Estados; en Anguila, dicho costo representó más de una vez el PIB (CEPAL, 2020, p. 62).

Los patrones de producción y consumo de la economía actual y que fueron profundizados con la globalización, son insostenibles por lo que se requiere cambiar la estrategia de desarrollo para desvincular el crecimiento de la contaminación por gases de efecto invernadero. La estrategia de desarrollo actual pone en riesgo la sobrevivencia del sistema ecológico que lo sustenta y los mercados no pueden frenar por sí mismo estos procesos, porque las tasas de rentabilidad no internalizan la destrucción de la naturaleza ni los efectos que esta tiene sobre la salud y el bienestar. El calentamiento global causado por la actividad humana es la expresión de la incapacidad del modelo económico para internalizar las variables ambientales.

\section{Una nueva estrategia para un cambio estructural}

América Latina y el Caribe aprovechó las tendencias favorables del contexto externo que motivaron la adopción del regionalismo abierto como estrategia de desarrollo (entre 1990 y 2008 , año de la crisis financiera mundial). Estas tendencias se expresaron en una mayor demanda internacional, un incremento en los precios de las materias primas, incrementos sostenidos del número de viajeros, un alto nivel de liquidez en los mercados financieros, y una creciente deslocalización productiva de la mano de la inversión extranjera directa. Entre 2000 y 2008, el crecimiento promedio de las exportaciones de la región fue del $4,5 \%$ y el de los flujos de IED hacia la región fue del 6,8\%. El producto interno bruto de la región registró estos impactos positivos, aunque de manera muy desigual en los países e insuficiente en la mayoría de ellos como para cerrar la brecha con los países desarrollados.

La crisis de 2008 marcó el fin de un ciclo expansivo y desde entonces, la región no logra un crecimiento sostenido, ni de su producto ni de su comercio. La inversión 
extranjera directa tampoco retoma los volúmenes que se registraron en la primera década del siglo. La productividad total de los factores muestra que la brecha de productividad entre América Latina y los Estados Unidos -este último como comparativo de alta productividad-entre 1960 y 2010 se incrementó de 26,6\% a 48,0\% (Fernández-Arias, Stein y Crespi, 2014). Las estimaciones recientes de la CEPAL muestran que la contribución de la productividad total de los factores al crecimiento de América Latina ha sido negativa a partir de 2012, lo que implicaría que la brecha con los países desarrollados se ha ampliado.

Hay que tener en cuenta que América Latina y el Caribe es la región más desigual del mundo y algunos de sus países presentan disparidades en la distribución del ingreso entre las más altas del mundo. El buen crecimiento de algunos países a principios de este siglo llevó a reducciones de la desigualdad, medida tanto por el índice de Gini como por índices complementarios como los de Theil y de Atkinson (con dos parámetros de aversión a la desigualdad, 1,0 y 1,5) (CEPAL, 2019). Sin embargo, diversos estudios que estiman la participación del percentil de mayores ingresos complementando la información de las encuestas de hogares con datos distributivos de los registros impositivos, muestran una persistente desigualdad de ingresos en la región. El correlato de la desigualdad está en la pobreza estructural de la región. En 2018, alrededor del 30,1\% de la población de América Latina estaba bajo la línea de pobreza, mientras que un 10,7\% se encontraba bajo el umbral de la pobreza extrema (CEPAL, 2019). Desde 2015 se observó un aumento en los niveles de pobreza, y especialmente de pobreza extrema, lo que tomó fuerza tras la pandemia de SARSCov2.

Por otra parte, los efectos del calentamiento global pasan una alta factura a la región y afectan de manera desproporcionada a los países con costas en el Caribe. En 2017 la temporada de huracanes tuvo un costo de 93.500 millones de dólares, y los daños superaron el producto interno bruto de algunas islas. Por su parte, la temporada de huracanes del Atlántico de 2020 fue la más activa de la historia y la quinta consecutiva en superar el promedio de actividad. Los eventos hidrometeorológicos han aumentado cada década desde 1950 y representan el $81 \%$ de los desastres de origen natural registrados en la región. Si bien se ha avanzado en la prevención, este tipo de desastres son responsables del $86 \%$ de las personas afectadas en la región, lo que pone en riesgo los avances logrados y la sostenibilidad del desarrollo. A estas catástrofes, se suman otros desastres que tienen efectos cumulativos, como los eventos de lento desarrollo como las sequías, y los de alta frecuencia y bajo impacto, como los deslaves e inundaciones causadas cada temporada lluviosa. En el Corredor Seco Centroamericano han convergido la sequía, la pandemia por SARSCov2 y la temporada de huracanes 
2020, provocando hambre en ocho millones de personas, frente a 2,2 millones en 2018 (Noticias, ONU, 2021).

La crisis sanitaria del SARSCov2 evidenció problemas estructurales de la región que, si bien no tienen origen en la pandemia, esta los ha manifestado con mayor crudeza: problemas ambientales, económicos y sociales. Resolver estos tres problemas requiere de una nueva estrategia de desarrollo para la región, la que debería alinearse con una nueva estrategia global. Esto implica plantear una propuesta de crecimiento ambientalmente sostenible, y socialmente inclusiva. Estos equilibrios requieren un conjunto de políticas y una recomposición de las fuerzas económicas: nuevas industrias dinámicas, reducción del peso de industrias contaminantes, nuevas regulaciones comerciales -tanto las relacionadas con la huella de carbono como con sus aportes sociales-, el rol del Estado en la economía -regulaciones ambientales, apoyos a sectores esenciales, ingresos mínimos, seguridad social e igualdad de derechos.

Tras un periodo en el que el péndulo de las políticas económicas apuntó a la reducción del papel del Estado en la economía, sobre todo para alejarlo de la actividad productiva, así como a la liberalización comercial y desregulación de los mercados, ahora las políticas económicas varían hacia una economía keynesiana en la que el Estado tiene un rol director. Aún es pronto para saber cuál será el conjunto de políticas económicas que emergerán ni el rol del Estado en la economía. Es claro que dos fuerzas luchan por atraer el péndulo hacia sendos extremos. La economía política subyacente en el consenso de Washington subsiste y procura restaurar las políticas económicas que se diseñaron en los años 80 y 90 del siglo pasado. Por otro lado, algunos sostienen que se requiere mucho más Estado y mucha más regulación para reconducir la economía hacia los nuevos equilibrios que se señalaron.

Las nuevas estrategias de desarrollo apuntan a impulsar nuevos sectores productivos a través de políticas industriales sostenibles que desacoplen el crecimiento de una economía basada en carbono. El resurgimiento de la política industrial es un signo de los cambios a favor de un rol más activo del Estado en el fomento a las inversiones, privadas y públicas. Las políticas industriales sostenibles no solo son fundamentales para la vida en la tierra, sino que sirven como un programa de inversiones de largo plazo. El gran impulso ambiental, siguiendo la propuesta descrita por Rosestein-Rodan, va de la mano de una política industrial basada en un keynesianismo ambiental que complementa los objetivos de empleo y cuidado ambiental con inversiones y crecimiento bajos en carbono (Altenburg y Rodrik, 2017; CEPAL, 2016). Esto está a la base de los esfuerzos recientes de grandes 
economías como la de los Estados Unidos y la Unión Europea que intentan cambiar sus matrices de producción y consumo para que sean más sostenibles.

Además de una agenda ambiental, América Latina y el Caribe debe enfrenar sus profundas desigualdades que se expresan en el ingreso de sus habitantes, pero también en materia de derechos y ciudadanía, en el acceso a servicios públicos y privados, y a la exposición a los riesgos de desastres. Una nueva agenda de desarrollo debe tener por meta la igualdad. La transformación productiva propuesta por las nuevas estrategias de desarrollo debe aspirar a una sociedad más igualitaria, en la que todas las personas, por el solo hecho de ser parte de la sociedad e independientemente de sus logros individuales, acceda a ciertos umbrales de bienestar social (Bárcena, et al, 2018).

El crecimiento económico sostenibley con mayor igualdad sirven de guía a los pactos sociales que están a la base de las nuevas políticas de desarrollo. Se vislumbran entonces debates y acuerdos en materia fiscal que contemplen una estructura y una carga tributaria con mayor efecto redistributivo, que diseñen nuevos papeles al Estado, así como una política pública que tenga como meta mejores umbrales de bienestar. Consecuentemente, a nivel global, podrían darse acuerdos mínimos sobre fiscalidad y tratamiento a las empresas transnacionales.

El cambio que se asoma en el política de desarrollo en América Latina y el Caribe podría mover el péndulo que ha estado durante más de 30 años en el ángulo de políticas derivadas del consenso de Washington y del regionalismo abierto, hacia el fortalecimiento de instituciones económicas, sociales y públicas que aseguren el acceso a los factores de la producción, una mejor protección social y distribución de los frutos de las innovaciones y de los incrementos de productividad, y unas regulaciones ambientales que promuevan una descarbonización del crecimiento económico.

\section{Reflexiones finales}

La búsqueda del desarrollo ha llevado a América Latina y el Caribe a implementar diversas estrategias que han tenido éxitos parciales. Es importante valorar esos éxitos con miras a que nuevas estrategias no partan de cero en el diseño de políticas económicas, al tiempo que se deben comprender los problemas estructurales no resueltos aún. 
En este artículo se ha señalado que existen claras señales de agotamiento de la estrategia de desarrollo basada en un crecimiento carbonizado, de plena liberalización y con fuertes reducciones del Estado. Aunque no está claro cuál será la estrategia de desarrollo emergente, algunos de los problemas estructurales que deben ser resueltos son cada vez más evidentes y gozan del creciente acuerdo internacional: sostenibilidad ambiental, igualdad e inclusión, crecimiento de la producción y de la productividad desacoplados de la economía del carbono.

Las propuestas de política económica que procuren el cambio estructural progresivo que se requiere pueden echar mano de los mercados más cercanos e integrados lo que conduciría al fortalecimiento de los procesos de integración; también requiere de incrementos en la capacidad de innovación para responder a los cambios necesarios en los patrones productivos; requiere de pactos fiscales nacionales e internacionales; necesita políticas sociales que procuren la igualdad de derechos. Por esta razón las políticas propuestas procurarían la complementariedad entre la innovación, el empleo, la distribución del ingreso y el crecimiento económico sostenible.

\section{Bibliografía}

AltenBURG, T. y RodRIK, D. (2017). "Green industrial policy: Accelerating structural change towards wealthy green economies", en AlteNBURG, T. \& AsSMANN, C. (Eds.), Green Industrial Policy. Concept, Policies, Country Experiences, UN Environment; German Development Institute / Deutsches Institut für Entwicklungspolitk (DIE), Ginebra y Bonn.

BANCOMUNDIAL (2021). Databank; https://databank.bancomundial.org/home.aspx

Bárcena, A., Bielschowsky, R. y Torres, M. (2018). "El séptimo decenio de la CEPAL: una reseña de su producción intelectual", en Desarrollo e igualdad: el pensamiento de la CEPAL en su séptimo decenio. Textos seleccionados del período 2008-2018, Colección 70 años, $n^{\circ} 1$ (LC/PUB.2018/7-P), R. Bielschowsky y M. Torres (comps.), Santiago, Comisión Económica para América Latina y el Caribe (CEPAL).

Bulmer-Thomas, V. (2003). La historia económica de América Latina desde la independencia, México: Fondo de Cultura Económica.

CEPAL (Comisión Económica para AmérICa Latina y el Caribe) (2021). https://estadisticas.cepal.org/cepalstat/portada.html 
- (2020). Construir un nuevo futuro: una recuperación transformadora con igualdad y sostenibilidad (LC/SES.38/3-P), Santiago.

- (2019). Panorama Social de América Latina, 2019 (LC/PUB.2019/22-P/Re v. 1), Santiago.

- (2016). Horizontes 2030: la igualdad en el centro del desarrollo sostenible (LC/G.2660/Rev.1), Santiago, julio.

- (2014). Cambio estructural para la igualdad: una visión integrada del desarrollo (LC/G.2524(SES.34/3)), Santiago, julio.

- (2012). Cambio estructural para la igualdad: una visión integrada del desarrollo. Sintesis (LC/G.2525(SES.34/4)), Santiago, agosto.

- (1994). El regionalismo abierto en América Latina y el Caribe: la integración económica al servicio de la transformación productiva con equidad, Libros de la CEPAL, $\mathrm{n}^{\circ} 39$ (LC/G.1801/Rev.1), Santiago, septiembre.

Cepal (Comisión Económica para América latina y el Caribe) y otros (2015). Cambio climático en Centroamérica: impactos potenciales y opciones de política pública (LC/MEX/L.1 196), Ciudad de México, noviembre.

Comisión EUROPEA (2014). Innovation Union Competitiveness Report, Dirección General para la Investigación y la Innovación, Bruselas.

CONTIPELI, E. (2016). "La Comunidad Andina de Naciones y la evolución del proceso de integración socioeconómico en Latinoamérica", en Estudios de Deusto, vol. 64, $n^{\circ}$ 1, 261-280; en línea: https://revista-estudios.revistas.deusto.es/issue/view/190

DASGUPTA, P. (2021). "The Economics of Biodiversity", The Dasgupta Review, London: HM Treasury.

Fernández-AriAs, E., Stein, E. y Crespl, G. (eds.) (2014). Rethinking Productive Development. Sound Policies and Institutions for Economic Transformation, InterAmerican Development Bank (IADB).

Martínez Piva, J. M. (editor) (2019). Logros y desafíos de la integración centroamericana: aportes de la CEPAL, Libros de la CEPAL, $n^{\circ} 156$ (LC/PUB.2019/7-P), Santiago de Chile. 
- (2015). "Política industrial en el sigloXXI", en Estrategias empresariales, política industrial y competitividad en las mipymes, M. LóPEz (coord.), Ciudad de México, Pearson.

- (2001). "El desarrollo local en América Latina", en Revista de Comercio Exterior, vol. 51, $\mathrm{n}^{\circ}$ 8, Ciudad de México, Banco Nacional de Comercio Exterior (Bancomext), agosto.

Moreno-Brid, J. C., Pérez-Caldentey, E. y Ruiz-Nápoles, P. (2004). "The Washington consensus: a Latin American perspective fifteen years later", Journal of Post Keynesian Economics, 27 (2), 345-365.

NoRDHAUS, W. (1994). Managing the global commons. The economics of climate change; MIT Press.

Noticias ONU (2021). "El clima y el COVID-19 cuadriplican el número de centroamericanos que pasan hambre", febrero, https://news.un.org/es/ story/2021/02/1488572

Organización Mundial de Comercio (OMC) (2021). "Regional Trade Agreements Database", en línea, https://rtais.wto.org/

Ortiz, G. (2003). "Latin America and the Washington Consensus. Overcoming Reform Fatigue", Finance \& Development, septiembre, Washington D.C.

Padilla-Pérez, R. y Martínez-PIVA, J. M. (2009). "Export growth, foreign direct investment and technological capability building under the maquila model: winding roads, few intersections", Science and Public Policy, vol. 36, $\mathrm{n}^{\circ}$ 4, mayo.

PINTO, A. (1970). "Naturaleza e implicaciones de la "heterogeneidad estructural" de América Latina", Trimestre Económico, vol. 37(1), n 145 (1), 83-100, México, Fondo de Cultura Económico.

Sánchez Díez, A. y Martínez PIVA, J. (2014). "Centroamérica: żuna nueva relación centro-periferia basada en el control de los activos productivos?", en Documentos de Proyectos (LC/W.578), Comisión Económica para América Latina y el Caribe (CEPAL), marzo, Santiago de Chile.

SteRn, N. (2007). "The Economics of Climate Change", The Stern Review, Cambridge University Press, Cambridge, Reino Unido. 
SUNKEL, O. (1991). "Del desarrollo hacia adentro al desarrollo desde dentro", en O. SUNKEL (Ed.), El desarrollo desde dentro. Un enfoque neoestructuralista para la América Latina, 35-80. México: Fondo de Cultura Económica.

UNCTAD (2021). UNCTADSTAT, hittps://unctadstat.unctad.org/ 